\title{
Review: selective serotonin reuptake inhibitors as effective as tricyclic antidepressants for major depression, and may have fewer adverse effects
}

MacGillivray S, Arroll B, Hatcher $S$ et al. Efficacy and tolerability of selective serotonin reuptake inhibitors compared tricyclic antidepressants in depression treated in primary care: systematic review and meta-analysis. BMJ 2003 May; 326:1014-20.

\section{QUESTION: How effective and safe are selective serotonin reuptake inhibitors compared with tricyclic antidepressant in people with depression treated in primary care?}

\section{Design}

Systematic review and meta-analysis.

\section{Data sources}

The reviewers searched the Cochrane Collaboration's depression, anxiety and neurosis group trials register to April 2002. Reference lists were hand searched and authors contacted

\section{Study selection}

Eligible studies were randomised controlled trials, comparing selective serotonin reuptake inhibitors versus tricyclic antidepressants in adults (aged 18-70 years) with depressive disorders who were being treated by a primary care practitioner. Exclusion criteria were: trials of treatment in a secondary or tertiary care setting and studies mainly in children or elderly people.

\section{Data extraction}

Methodological quality was independently assessed by two reviewers. Data were extracted on: trial participants' age, gender and diagnosis; drug and dose; study setting; duration of study and results. The primary outcomes were efficacy measured using standardised weighted mean differences (SWMD) of the final mean depression scores and relative risk of improvement on the Clinical Global Impression score. The review also examined withdrawals from treatment because of adverse effects.

\section{Main results}

Included studies: 11 studies (2954 participants, mean age 40.5 years, $75 \%$ female) were included. Severity of depression varied among participants. 4 of these studies were of low quality and were analysed separately in the meta-analysis.

Efficacy: 6 studies (2295 people) reported on efficacy outcomes. Selective serotonin reuptake inhibitors and tricyclic antidepressants were equally effective in the treatment of people with depression in primary care. (SWMD of final mean continuous depression scale scores $0.07,95 \% \mathrm{CI}-0.02$ to +0.15 ). Data from 3 studies (740 people) using the Clinical Global Impression score showed no significant difference in improvement between the two treatments (relative risk 1.11 95\%CI 0.86 to 1.43 ).

Adverse effects: 7 studies (2567 people) reported on adverse effects of treatment. Withdrawal due to adverse effects was significantly more likely with tricyclic antidepressants than with selective serotonin reuptake inhibitors (relative risk1.37, 95\% CI 1.14 to $1.66, \mathrm{p}<0.001$ )

\section{Conclusions}

Selective serotonin reuptake inhibitors are better tolerated and as effective as tricyclic antidepressants for short term treatment of people with depression in the primary care setting. The reliability and generalisability of these conclusions is limited by the variable quality and inclusion criteria of the included studies.
Source of funding:

This work was funded by Chief Scientist

Office, Scotland, Health Services Research Committee.

For correspondence: S MacGillivra). Department of Epidemiology and Public Health,

Ninewells Hospital and Medical School, University of Dundee,

Dundee DD1 9SY.

E-mail:

s.a.macgilliwray@ dundee.ac.uk

\section{COMMENTARY}

The meta-analysis carried out by MacGillivray et al, investigating the efficacy and tolerability profile of antidepressants in general practice, raises some interesting methodological questions connected with the conduct of systematic reviews.

First, 11 randomised controlled trials met the inclusion criteria, but 4 of them were subsequently excluded because of low quality. This approach contrasts with the general principle of including all studies meeting inclusion criteria. Low-quality studies could have been included to generate evidence on the independent contribution of study quality on outcome. As it stands, it remains unclear whether their exclusion, based on debatable parameters (for example, inadequate withdrawal details) has hampered the comparison.

Second, the analysis included one three-arm trial comparing imipramine with two different dose regimens of citalopram (group one 10-30 mg/day and group two 20-60 $\mathrm{mg} /$ day). ${ }^{1}$ Authors included both imipramine in comparison with citalopram 10-30 and imipramine in comparison with citalopram 20-60, however, it seems from the forrest plots that the imipramine-treated patients were counted twice in the calculation of the overall efficacy and tolerability estimates.

Third, discrepancies exist among data extracted from this three-arm study by these authors, and the data reported in the original trial ${ }^{1}$ and in another systematic review. ${ }^{2}$ Additional information may have been obtained by the authors, but their sources are not transparent.

Finally, from another study, ${ }^{3}$ authors extrapolated 12-week scores from graphs even though in the text of the article (and in the graphs) end-point scores, based on a last observation carried forward analysis (which seems closer to the principles of the intention-to-treat analysis), were reported. This latter figure was considered in another systematic review. ${ }^{2}$

These observations are not merely academic, especially in a systematic review including only seven trials, as they may lead to differences in outcome. It is important that systematic reviews include all trials meeting inclusion criteria, and that pre-planned subgroup analyses be performed to investigate the influence of independent variables (setting, trial quality) on outcome.

Andrea Cipriani, MD Department of Medicine and Public Health, Section of Psychiatry University of Verona, Italy

1 Rosenberg C, Damsbo N, Fuglum E, Jacobsen LV, Horsgard S. Citalopram and imipramine in the treatment of depressive patients in general-practice. A Nordic multicentre clinical in the treatment of depressive patients in general-p

2 Ceddes JR Frente indes JR, Freenate for N, Mason J, Eccles MP, Boynton J. Selective serotonin reuptake 2003. Oxford: Update Software.

Ravindran AV, Judge R, Hunter BN, Bray J, Morton NH. A double-blind, multicentre study in primary care comparing paroxetine and clomipramine in patients with depression and in primary care comparing paroxetine and clomipramine in patients with depre 